\title{
$\beta$-catenin induces expression of prohibitin gene in acute leukemic cells
}

\author{
DONG MIN KIM ${ }^{1}$, HANBIT JANG ${ }^{2}$, MYUNG GEUN SHIN ${ }^{3}$, JEONG-HOON KIM ${ }^{2}$, \\ SANG MO SHIN ${ }^{1}$, SANG-HYUN MIN ${ }^{4}$ and IL-CHUL KIM ${ }^{5}$ \\ ${ }^{1}$ Center for Applied Life Science, Hanbat National University, Daejon 305-719; ${ }^{2}$ Medical Proteomics Research Center, \\ KRIBB, Daejon 305-806; ${ }^{3}$ Department of Laboratory Medicine, Chonnam National University Hwasun Hospital, \\ Chonnam National University, Hwasun 519-763; ${ }^{4}$ New Drug Development Center, DGMIF, Daegu 701-310; \\ ${ }^{5}$ Department of Biological Sciences, Chonnam National University, Gwangju 500-757, Republic of Korea
}

Received November 2, 2016; Accepted April 3, 2017

DOI: $10.3892 /$ or.2017.5599

\begin{abstract}
Prohibitin (PHB) is a multifunctional protein conserved in eukaryotic systems and shows various expression levels in tumor cells. However, regulation of PHB is not clearly understood. Here, we focused on the regulation of $P H B$ expression by Wnt signaling, one of dominant regulatory signals in various leukemic cells. High mRNA levels of $P H B$ were found in half of clinical leukemia samples. PHB expression was increased by inhibition of the MAPK pathway and decreased by activation of EGF signal. Although cell proliferating signals downregulated the transcription of PHB, treatment with lithium chloride, an analog of the Wnt signal, induced PHB level in various cell types. We identified the TCF-4/LEF-1 binding motif, CATCTG, in the promoter region of $P H B$ by site-directed mutagenesis and ChIP assay. This $\beta$-catenin-mediated activation of $P H B$ expression was independent of c-MYC activation, a product of Wnt signaling. These data indicate that $P H B$ is a direct target of $\beta$-catenin and the increased level of PHB in leukemia can be regulated by Wnt signaling.
\end{abstract}

\section{Introduction}

Prohibitin 1 (PHB) is multifunctional protein and highly conserved among yeast, plants, worms, flies, and mammals (1-5). The eukaryotic mitochondrial PHB complex, which associates to form a ring-like structure, has a role as a holdase type of chaperone specifically required in situations of mitochondrial stress $(6,7)$. Despite much evidence that PHB is located at the mitochondrial inner membrane, various cellular functions

Correspondence to: Dr Sang-Hyun Min, New Drug Development Center, DGMIF, Daegu 701-310, Republic of Korea

E-mail:shmin03@gmail.com

Professor Il-Chul Kim, Department of Biological Sciences, Chonnam National University, Gwangju 500-757, Republic of Korea E-mail: ickim@chonnam.ac.kr

Key words: prohibitin, leukemia, $\beta$-catenin, c-MYC, PHB have been proposed for this protein outside the mitochondria. PHB was found at the plasma membrane of human intestinal epithelial cells where it functions as a binding site for the Vi capsular polysaccharide of Salmonella typhi (8). It has also been found to be the target for a proapoptotic peptide in adipose vasculature (9). Furthermore, it has been implicated in mediating cellular Ras-Raf signaling at the membrane (10). $\mathrm{PHB}$ also binds to a wide range of proteins including retinoblastoma protein, E2F transcription factor, Brg1/Brm and p53 $(11,12)$. In breast cancer cell lines, PHB co-localizes in the nucleus with E2F1, retinoblastoma protein and $\mathrm{p} 53$, and the interaction of $\mathrm{PHB}$ with $\mathrm{E} 2 \mathrm{~F} 1$ and retinoblastoma protein can be complex (13-15). The coiled-coil domain of PHB is important in the interaction of PHB with E2F1 and HDAC1 (16). Recent reports suggest that recruitment of Brg1/Brm to $\mathrm{E} 2 \mathrm{~F}$ responsive promoters is required for repression of E2F mediated transcription by PHB and involves the JNK pathway (17). $\mathrm{Brg} 1 / \mathrm{Brm}$ are involved in chromatin remodeling and has been implicated in transcriptional activation by the estrogen receptor (18). PHB was also shown to repress the activity of estrogen receptor $\alpha$ (19). These findings indicate that $\mathrm{PHB}$ has several roles in cell cycle progression, the regulation of transcription, and in cell surface signaling. PHBs are required for embryonic development and in tissues that undergo cellular proliferation (20).

Wide investigation of $P H B$ expression revealed that it is constitutively expressed in normal mammalian cells such as hepatocytes, smooth muscle cells, chondrocytes, spermatocytes and oocytes (21). Higher levels of $P H B$ expression were found in regenerating liver cells, chemically-induced carcinoma, hyperplastic hepatic nodules and hepatocellular carcinomas, and in cancer cells and primary tumor samples (7,22-24). The expression of $P H B$ increases in yeast cells during diauxic shift when cells switch from non-oxidative to oxidative metabolism (7). There are MYC-binding elements in the promoter region of $P H B$, and the $c-M Y C$ oncogene is overexpressed in many cancer cells (25). In a B-cell lymphoma line, overexpression of $P H B$ protects the cells from apoptosis, via regulation of E2F activity (26). Also retinoic acid or hexamethylene bisacetamide can stimulate the translocation of PHB to the nucleus in tumor cells (27). 
Among cancers, acute myeloid leukemia (AML) is a heterogeneous human disease and involves a multi-step process characterized by alteration in different pathways affecting cell proliferation and myeloid differentiation $(28,29)$. Although in chronic myeloid leukemia samples with different $\beta$-catenin levels lead to divergent effects in a Bcr-Abl model of leukemogenesis (30), no $\beta$-catenin mutations have been found in AML. Translocation products, such as AML1-ETO (eight twenty-one) and promyelocytic leukemia zinc finger-retinoic acid receptor $\alpha$ (PLZF-RAR $\alpha$ ) activate the Wnt pathway through plakoglobin activation (31). Siapati et al demonstrated the relevance of the Wnt pathway in AML cell lines (32).

In this study, we investigated Wnt-mediated PHB expression in leukemic samples containing ALL and AML, and found that Wnt signaling can activate transcription of $P H B$ via the TCF-4/LEF-1 binding motif in the promoter of $P H B$ in a MYC-independent manner. These data suggest that the elevated level of PHB in leukemic cells is the result of Wnt signals which are upregulated in leukemic cells.

\section{Materials and methods}

Cell culture and chemicals. HeLa cells were grown in DMEM (WelGene, Daegu, Korea) supplemented with $10 \%$ (v/v) fetal bovine serum (JR Scientific, Inc., Woodland, CA, USA), 1\% (v/v) penicillin/streptomycin (P4333, Sigma-Aldrich Korea, Seoul, Korea). Leukemic cells provided by patients and Raji cell from ATCC were grown in RPMI-1640 (WelGene) with same supplements as with DMEM. For inhibition of c-MYC binding to DNA, 10058-F4 ([Z,E]-5-[4-ethylbenzylidine]-2thioxothiazolidin-4-one, F3680, Sigma-Aldrich Korea) was dissolved in $0.5 \%$ (v/v) DMSO (D5879, Sigma-Aldrich Korea). Chemicals were administered to the cells in $1 \%$ serum condition and cells were incubated for 5-8 h.

Plasmid construction for promoter assay and site-directed mutagenesis. To quantify the transcription amount of $P H B$, we cloned the promoter region of PHB from genomic library of HeLa cells using primers, PHBF3 (5'-CTCAAGCTTTTCC AAATAAAA-3') and PHBR4 (5'-ACTGGATCCACATGA ATTCCC-3'). The amplified 650-bp fragment was treated with Klenow and BamHI and cloned into the pcDNA-luciferase plasmid. HeLa cells were transfected by the resulting PHB-Luc promoter reporter and the promoter activity was analyzed using a luciferase assay. To mutate the transcription binding sites in PHB promoter, the PCR product of the promoter region was subcloned into pGEM $^{\circledR}$-T Easy Vector system (A1360, Promega Korea, Seoul, Korea) and site-directed mutations were introduced using the QuickChange ${ }^{\circledR}$ Site-Directed Mutagenesis method (Agilent Technologies, Inc., Wilmington, DE, USA). To remove candidate sites for TCF- 4 binding to the promoter, the following primers were used; PHBm1F (5'-GTT GTAGTTCAGATGCTAAGAGCC-3') and PHBm1R (5'-GGC TCTTAGCATCTGAACTACAAC-3'); PHBm2F (5-GGAA GCGTGGAGATTGGAAAGCGG-3') and PHBm2R (5'-CCG CTTTCCAATCTCCACGCTTCC-3'). To mutate the binding site of c-MYC, the following primers were used; PHBm4F (5'-TACAGGATAGGCATATGCATTTAGCCCC-3') and PHBm4R (5'-GGGGCTAAATGCATATGCCTATCCT GTA-3'). After PCR reaction with PHB promoter in T-vector and $D p n I$-digestion, the transformants were identified by sequencing. After identification of mutated sequences, the promoter regions were subcloned into the PHB-Luc plasmid by exchange of the promoter region digested with $\mathrm{XhoI}$ and BamHI restriction enzymes.

Determination of RNA amounts. Total RNAs from cells were extracted with TRIzol ${ }^{\mathrm{TM}}$ (Invitrogen) and first-strand cDNAs were synthesized using AMV reverse transcriptase (F-570S, Finzymes, Espoo, Finland) at $42^{\circ} \mathrm{C}$ for $1 \mathrm{~h}$. For real-time PCR, the first-strand cDNA mixtures corresponding to $5 \mu \mathrm{g}$ of total RNA served as templates for PCR analysis with the SYBR $^{\circledR}$ premix Ex Taq ${ }^{\mathrm{TM}}$ (Takara, Kyoto, Japan). Reactions were conducted on a Corbett RG-6000 apparatus (Corbett Life Science, New South Wales, Australia). To optimize the PCR conditions in terms of primers, annealing temperatures, PCR efficiency, and standard curves were used. The PCR conditions were as follows: cycles of $95^{\circ} \mathrm{C}$ for $10 \mathrm{sec}, 58^{\circ} \mathrm{C}$ for $10 \mathrm{sec}$ and $72^{\circ} \mathrm{C}$ for $15 \mathrm{sec}$. To calculate the relative expression levels of genes of interest, the $\mathrm{Ct}$ value of GAPDH (glyceraldehyde-3-phosphate dehydrogenase) expression was used as a normalizer. Amplification of cDNAs was performed using primers: GDH1 (5'-TGAGAACGGGAAGCTTGTCA-3') and GDH2 (5'-GGAAGGCCATGCCAGTGA-3'); PHBF1 (5'-TCA TTTTCTCATCCCGTGGGTA-3') and PHBR1 (5'-TGCCTGG AGACCAGCTCTCT-3'); MYCF1 (5'-CGTCTCAGAGAAGC TGGCCT-3') and MYCR1 (5'-TGGCCTCCAGCAGAAGG TGA-3'); CCND1F (5'-GGCGGAGGAGAACAAACAGA-3') and CCND1R (5'-TGGCACAGAGGGCAACGA-3').

Immunoblot assay. Cultivated cells were harvested and lysed using lysis buffer [1\% (v/v) Triton X-100, $200 \mathrm{mM}$ HEPES (pH 7.9), $300 \mathrm{mM} \mathrm{NaCl}, 100 \mathrm{mM} \mathrm{KCl}, 10 \mathrm{mM}$ EDTA, $10 \mu \mathrm{g} / \mathrm{ml}$ aprotinin, $100 \mu \mathrm{g} / \mathrm{ml}$ leupeptin, and $10 \mu \mathrm{M}$ PMSF]. Twenty micrograms of total protein lysate was electrophoresed in an acrylamide gel and transferred to a TransBlot ${ }^{\circledR}$ membrane (162-0145, Bio-Rad, Hercules, CA, USA). Membranes were blocked in $5 \%(\mathrm{w} / \mathrm{v})$ milk, incubated with primary antibodies for $1 \mathrm{~h}$ at room temperature, washed and incubated for $1 \mathrm{~h}$ with the appropriate HRP-conjugated secondary antibodies (sc-2004 and sc2005, Santa Cruz Biotechnology, Inc., Santa Cruz, CA, USA). The blots were washed in PBS containing $0.05 \%$ (v/v) Tween-20 ${ }^{\circledR}$ (P1379, Sigma-Aldrich Korea) and an ECL kit (34080, Pierce Biotechnology, Rockford, IL, USA) was used prior to detection of protein bands. The antibodies used were: rabbit anti-prohibitin (sc-28259, Santa Cruz Biotechnology, Inc.), rabbit anti- $\beta$-catenin (sc-7199, Santa Cruz Biotechnology, Inc.), rabbit anti-GSK-3 $\beta$ (sc-9166, Santa Cruz Biotechnology, Inc.), rabbit anti-p-GSK-3 $\beta$ (sc-11757-R, Santa Cruz Biotechnology, Inc.), mouse anti-c-Myc (sc-40, Santa Cruz Biotechnology, Inc.), and mouse anti- $\beta$-actin monoclonal (sc-8432, Santa Cruz Biotechnology, Inc.) to determine equal loading.

Promoter analysis. For the luciferase reporter assay, $0.4 \mu \mathrm{g}$ of PHB-Luc and $0.2 \mu \mathrm{g}$ of CMV-LacZ were cotransfected into HeLa cells in 6-well plates (Corning Inc., Corning, NY, USA) using the HilyMax Transfection reagent (Dojindo, Kumamoto, Japan). Luciferase assay was performed $48 \mathrm{~h}$ after transfection using a Luciferase assay system (E1501, Promega Korea) 
A
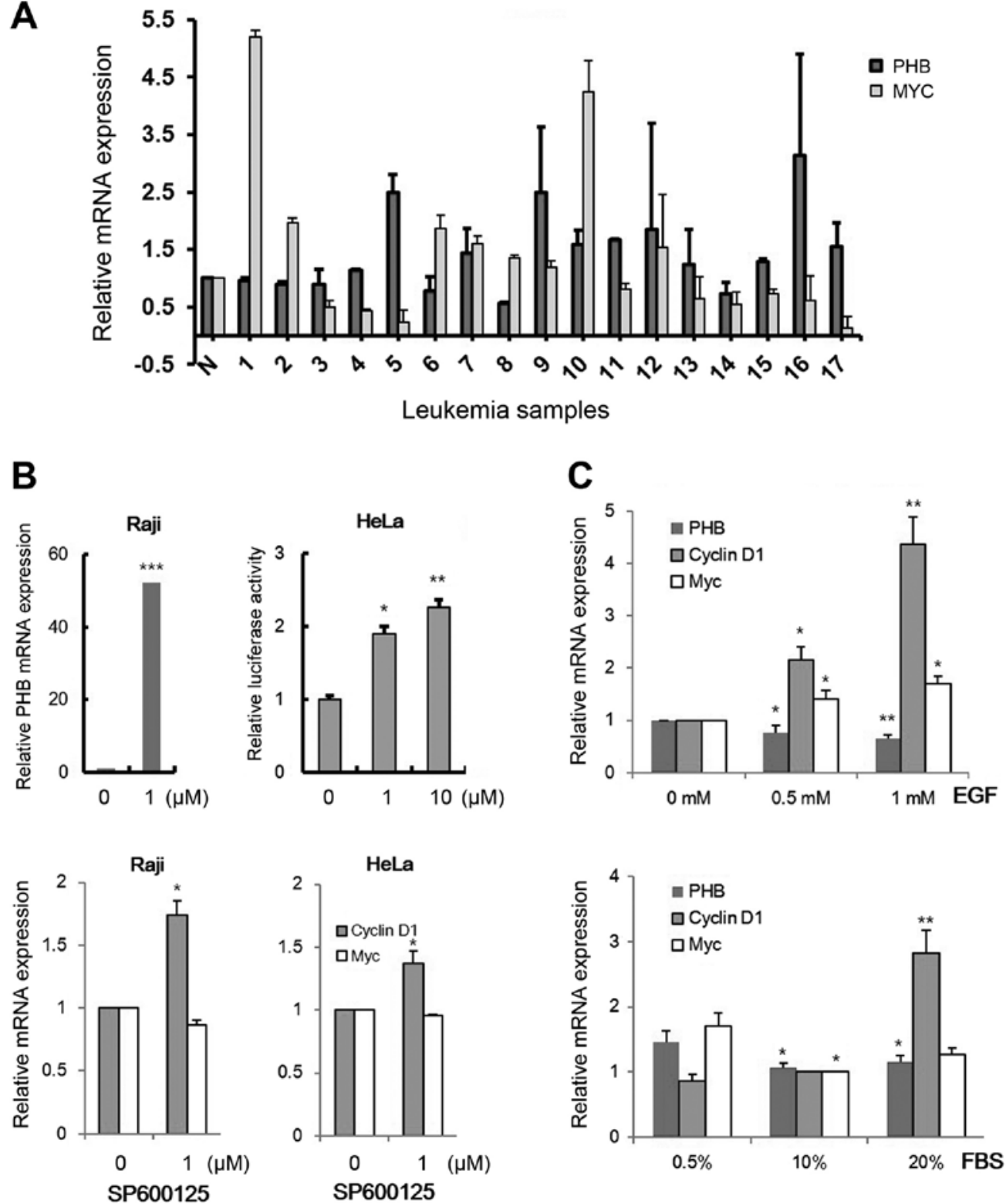

Figure 1. Expression of PHB and c-Myc in acute leukemia samples. (A) Relative mRNA levels of PHB and c-MYC in 17 leukemia samples. N is average value of 4 normal samples and the real-time PCR analysis was performed by three times. Black and white bars represent the relative mRNA levels of PHB and c-MYC, respectively. (B) The effect of blocking MAPK pathway by SP600125 on PHB expression in Raji cells (left panel) by real-time PCR and HeLa (right panel) cells by reporter assay with PHB-Luc (WT). (C) Effects of growth signals on the expression of PHB in HeLa cells. After treatment with EGF (left panel) or various concentration of fetal bovine serum (right panel) on HeLa cells, the mRNA levels of PHB were monitored by real-time PCR. Data are presented as mean $\pm \mathrm{SD}(\mathrm{n}=3) .{ }^{*} \mathrm{p}<0.05,{ }^{* *} \mathrm{p}<0.01$ and ${ }^{* * *} \mathrm{p}<0.001$.

and a Lumat LB9501 instrument (Berthold, Bad Wildbad, Germany). For normalization, the $\beta$-galactosidase activity in transfected cells was determined spectrophotometrically at $420 \mathrm{~nm}$ using $0.2 \%$ (w/v) 2-nitrophenyl $\beta$-D-galactopyranoside (N1127, Sigma-Aldrich Korea). Transfections were performed in duplicate and repeated a minimum of three times.

The binding of transcription factors to promoter region of the $P H B$ was monitored using a ChIP assay kit (Upstate Biotechnology, Lake Placid, NY, USA). After cultivation of Raji cells in a 100-mm dish (Corning, Inc.) with chemicals, $37 \%$ formaldehyde was added to the culture media for $10 \mathrm{~min}$ to cross-link DNA and proteins. After washing the cells with PBS, cell pellets were resuspended in lysis buffer from the kit with protease inhibitors. The cells were sonicated and the supernatants were used in the ChIP assay according to the manufacturer's protocol. The fragmented chromatin was pulled-down using a rabbit anti- $\beta$-catenin antibody (sc-7199, Santa Cruz Biotechnology, Inc.; 1:2,000) and 163- and 250-bp products were amplified with primers: PHBpF (5'-CCGCC GCTGCATAGCCTTT-3') and PHBpR (5'-TGCGCATGAGC TCCTCTGC-3')for PHB; CCND1PF( 5 '-AGGCGCGGCGCCT CAGGC-3') and CCND1PR (5'-TGGAGGCTCCAGGACT TTGCA-3') for the cyclin D1 gene, respectively.

\section{Results}

High-levels of $P H B$ expression in leukemic cells. It was reported that $P H B$ is one of the targets of c-MYC, and an 
A

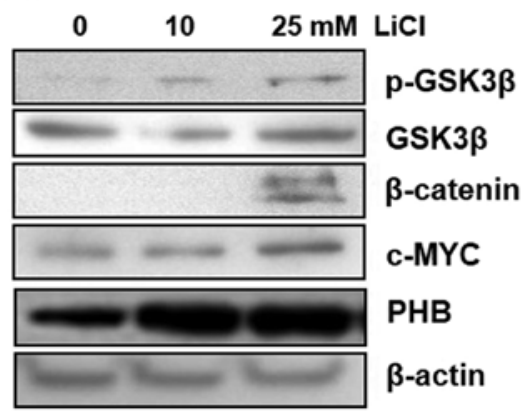

B

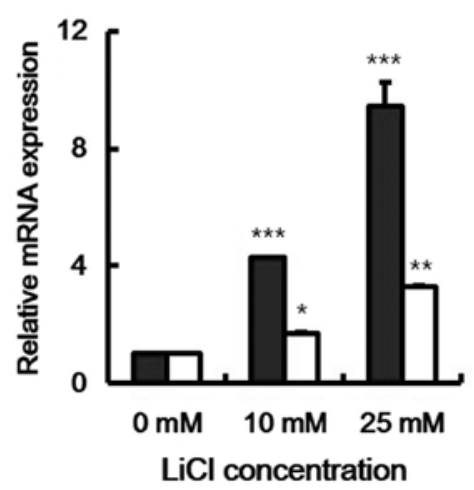

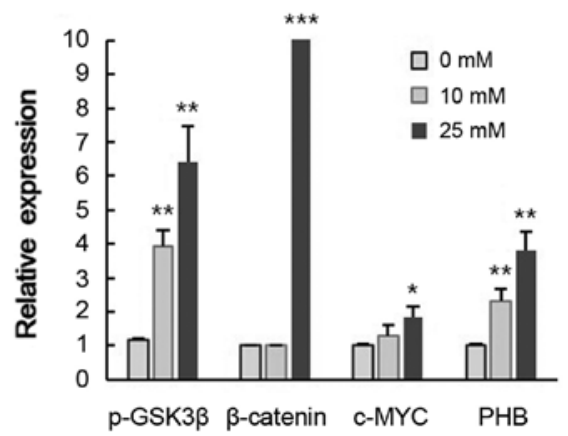

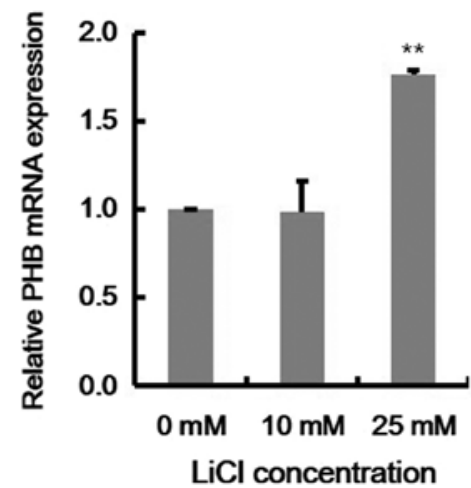

Figure 2. Induction of PHB expression by Wnt signaling in leukemic cells. (A) Activation of Wnt signaling and increased PHB expression by LiCl in Raji cells. After treatment with $\mathrm{LiCl}$ for $48 \mathrm{~h}$ in Raji cells, protein levels were analyzed by immunoblot assay and $\beta$-actin levels were used as a normalizer. (B) Effect of Wnt signaling on the expression of PHB and Wnt target genes in Raji cells. After treatment with LiCl for $48 \mathrm{~h}$ in Raji cells, the mRNA levels of cyclin D1 (black bars in left panel), c-MYC (white bars in left panel), and PHB (right panel) were analyzed by real-time PCR using the mRNA level of GAPDH as a normalizer. The PCR analysis was performed three times. Data are presented as mean $\pm \operatorname{SD}(\mathrm{n}=3) .{ }^{*} \mathrm{p}<0.05,{ }^{* *} \mathrm{p}<0.01$ and ${ }^{* * * *} \mathrm{p}<0.001$.

oncogenic transcription factor (19). Since $P H B$ expressions was increased in several acute and chronic myeloid leukemic cells in our preliminary study, we checked the relationship between $c-M Y C$ and $P H B$ expression patterns in clinical leukemic cells (Fig. 1A). Increased $P H B$ transcripts were detected in 9 samples $(5,7,9,10,11,12,13,16$ and 17) among 17 clinical samples (52.9\%) and the enhanced levels of both $c-M Y C$ and $P H B$ expression were observed in only 3 samples, which are sample numbers 7, 9 and $10(17.6 \%)$. We also confirmed $P H B$ expression by cell growth-promoting signals activating c-MYC activity (33). To screen a cell line expressing low level of PHB, we tested 15 leukemic cells, selected the Raji cell line (data not shown) and checked the transcriptional change in PHB by several oncogenic signals (Fig. 1B and C). PHB has been known to be associated with mitochondria function and JNK-1 reduces mitochondria content via promoting the nuclear localization of DAF-16/FOXO complex (34). Moreover, JNK regulates the protein stability of c-Myc which is a transcription factor to induce PHB transcription $(35,36)$. Therefore we tested whether JNK inhibitor regulates PHB expression. The $P H B$ mRNA level was increased by treatment with $1 \mu \mathrm{M}$ of SP600125, a JNK inhibitor, in Raji cells (Fig. 1B, left panel). This regulation was also detected in HeLa cells using a promoter-reporter system (PHB-Luc WT) after transfection (Fig. 1B, right panel). EGF ligand and serum slightly decreased PHB transcription in HeLa cells (Fig. 1C). These results showed that other factors but not routine signals for cell growth could exist to activate $P H B$ expression in leukemic cells.

Wnt signaling enhances PHB expression in leukemic cells. Because accumulation of $\beta$-catenin in the nucleus has been identified in chronic and acute leukemia $(37,38)$, we investigated the effect of $\beta$-catenin accumulation on PHB expression. We monitored the expression levels of $P H B$ with lithium chloride $(\mathrm{LiCl})$ treatment, which blocks GSK-3 $\beta$ activity and ultimately affects $\beta$-catenin accumulation (39). After treatment Raji cells with $\mathrm{LiCl}$, components of the Wnt signaling pathway were checked by immunoblot assay. With $\mathrm{LiCl}$, GSK-3 $\beta$ was phosphorylated while $\beta$-catenin, c-MYC and PHB protein levels were increased (Fig. 2A). The transcriptional levels of $\beta$-catenin target genes, $c-M Y C$ and $C C N D 1$, were increased (Fig. 2B, left panel), and also at $25 \mathrm{mM} \mathrm{LiCl}$, transcription of $P H B$ was significantly increased by 2 -fold (Fig. 2B, right panel). These data indicate that PHB expression was induced by Wnt signaling in leukemic cells.

TCF-4/LEF-1 binds to the PHB promoter. Because PHB expression was responsive to Wnt signaling via $\beta$-catenin, we searched for TCF-4/LEF-1 binding sequences in the promoter region of $P H B$. From information in the Transcriptional Regulatory Element Database (TRED:http:// rulai.cshl.edu/cgi-bin/TRED/tred.cgi?process=home), two candidate sequences were found (Fig. 3A). In contrast to the 

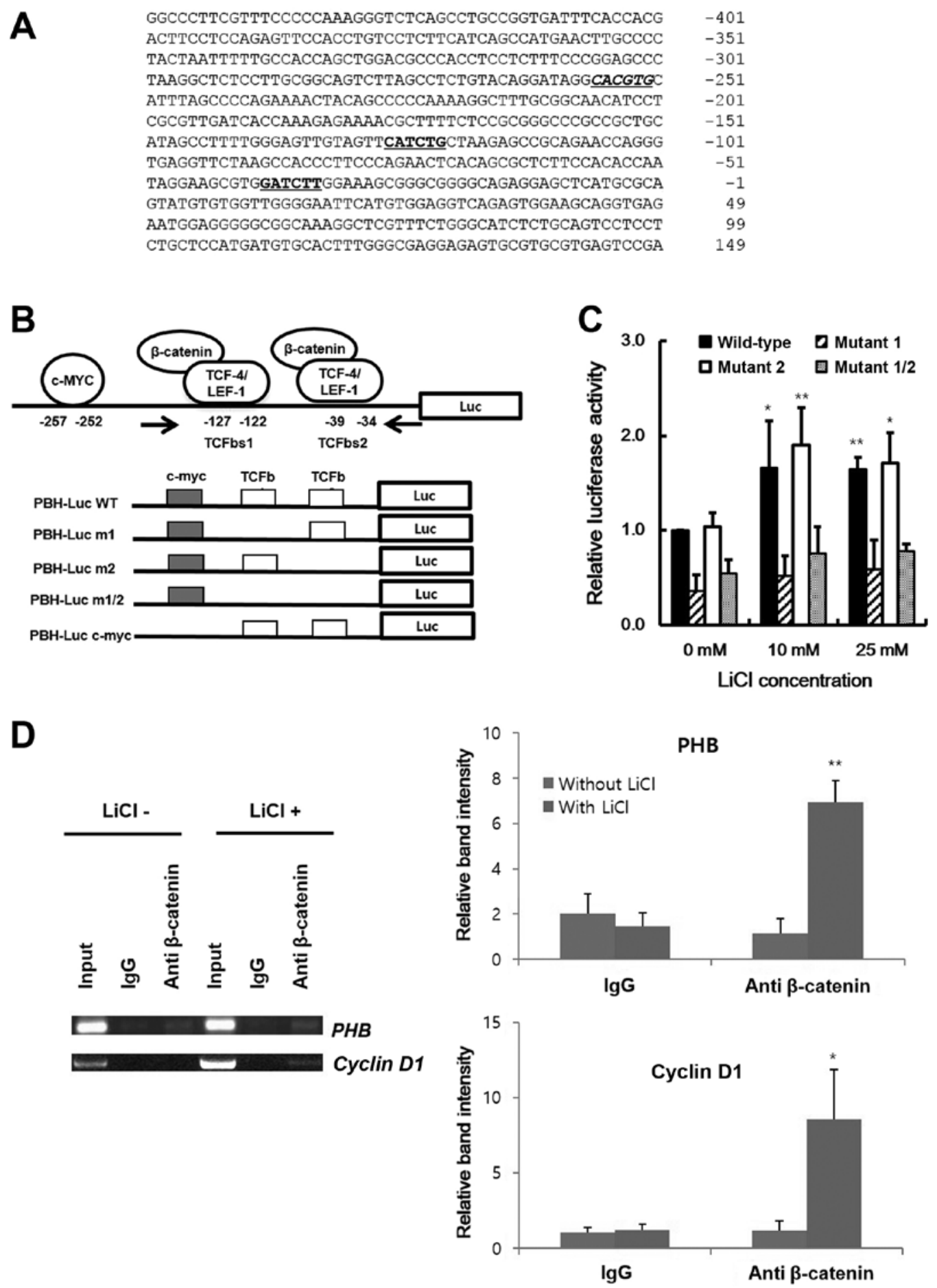

Figure 3. Enhancement of PHB transcription by activated $\beta$-catenin. (A) The nucleotide sequence of PHB promoter of human. The candidate c-MYC and TCF-4/LEF-1 binding sites are indicated by italics and underlined sequences, respectively. The numbering starts from the transcription start site as +1 . (B) Schematic representation of the cloned promoter region of PHB to construct the reporter vector (PHB-Luc) and its mutants. The candidate sites of TCF-4/LEF-1 are represented by TCFbs1 and TCFbs2, and the primers for identification of the pulled-down genomic fragments in the chromatin immunoprecipitation are indicated by arrows. The mutant $\mathrm{m} 1$ and $\mathrm{m} 2$ contain the modified TCFbss at -127 to -122 and -39 to -34 in promoter region, respectively. The double mutant $\mathrm{m} 1 / 2$ contains the modified sequences at both sites. (C) Identification of TCF-4/LEF-1 binding sequence in PHB promoter. After treatment of $\mathrm{LiCl}$ for $24 \mathrm{~h}$ in HeLa cells transfected with PHB-Luc or its mutants, the emitted luminescence was monitored with a luminometer. Black, diagonal, white and gray bars represent the relative activity of PHB-Luc promoter reporter of wild-type, $\mathrm{m} 1, \mathrm{~m} 2$ and double mutant $\mathrm{m} 1 / 2$, respectively. (D) Binding of $\beta$-catenin on PHB promoter. After chromatin immunoprecipitation using genomic DNA from Raji cells by anti- $\beta$-catenin antibody, the candidate regions were amplified with the PCR primer set in (B). The cyclin D1 promoter region was used as a positive control. Data are presented as mean \pm SD $(n=3)$. ${ }^{*} p<0.05,{ }^{* * *} p<0.01$ and ${ }^{* * * *} \mathrm{p}<0.001$.

identified TCF-4/LEF-1 binding motives, GCTTTGATCTT or GCCATCTG, these sites have a half-conserved sequence. TCFbs1, between -127 and -122 , is CATCTG and TCFbs2, between -39 and -34 , is GATCTT. To confirm TCF-4/LEF-1 binding on the PHB promoter, we cloned the promoter region in a luciferase reporter vector (Fig. 3B). With $\mathrm{LiCl}$ treatment in 
A

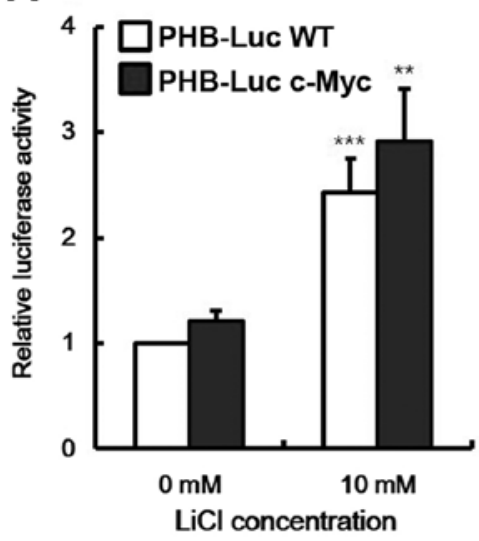

B

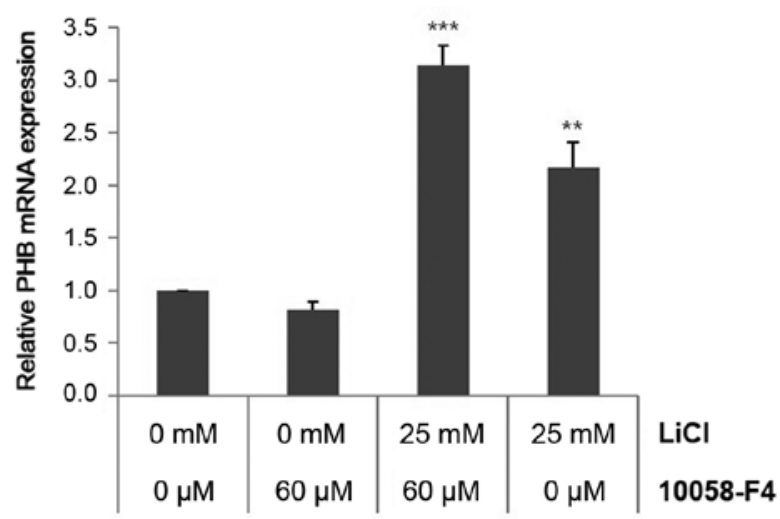

Figure 4. MYC-independent activation of PHB expression by Wnt signal. (A) Effects of c-MYC on the PHB-Luc promoter reporter in HeLa cells. The reporter assay using PHB-Luc with a mutation in the c-MYC binding site (PHB-Luc c-myc) was performed by three times (white bars, PHB-Luc WT; black bars, PHB-Luc c-Myc). (B) Effects of c-MYC inhibition on PHB expression in Raji cells. After treatment of $60 \mu \mathrm{M}$ of $10058-\mathrm{F} 4$ and $10 \mathrm{mM}$ of LiCl for $24 \mathrm{~h}$, the mRNA level of PHB was relatively quantified by real-time PCR and the GAPDH level was used as a normalizer. Data are presented as mean \pm SD ( $=3$ ). ${ }^{*} \mathrm{p}<0.05,{ }^{* *} \mathrm{p}<0.01$ and ${ }^{* * *} \mathrm{p}<0.001$.

HeLa cell, wild-type of PHB promoter activity was increased (Fig. 3C). To identify the active site of $\beta$-catenin in promoter region, the nucleotide sequences of the $\mathrm{PHB}$ promoter were changed by site-directed mutagenesis (Fig. 3B). In PHB pm1, CATCTG was changed to CAGATG, and GATCTT to GAGATT in PHB pm2. In the absence of $\mathrm{LiCl}$, the activity of PHB-Luc pm1 reporter was decreased by 2-fold compared with that of PHB-Luc wild-type (WT) promoter. Also, in the presence of $\mathrm{LiCl}$, activities of PHB-Luc pm1 and pm1/2 reporter were not increased whereas wild-type and pm2 PBH-Luc activities were increased (Fig. 3C). These results indicated that TCFbs1 is the $\beta$-catenin-working site and $P H B$ is a direct target of Wnt signaling. To confirm $\beta$-catenin binding in this region, using primers covering these sites (Fig. 3B, arrows), the binding of $\beta$-catenin to this region was checked by a chromatin immunoprecipitation assay in Raji cells. Fig. 3D indicated that with $\mathrm{LiCl}$, the accumulated $\beta$-catenin bound to this region through the TCF-4/LEF-1 binding sequence (TCFbs1) and $P H B$ was one of the direct Wnt target genes. Although the region pulled down by anti- $\beta$-catenin antibody was amplified in the absence of $\mathrm{LiCl}$, due to a basal level of $\beta$-catenin in the Raji cells, the amount of PCR product of this region was increased by $\mathrm{LiCl}$ treatment. These data suggest that TCFbs1 is a ubiquitous working site of $\beta$-catenin and $P H B$ is a direct target gene of Wnt signaling.

Effects of $c-M Y C$ and $\beta$-catenin on PHB expression. Because $c-M Y C$ is a known target gene of Wnt signaling and it can activate $P H B$ expression (25), we checked the dependency of c-MYC on $P H B$ transcription from Wnt signaling. Despite removal of the c-MYC binding site ( -257 to -252 in Fig. 3A and $\mathrm{B}), \mathrm{CACGTG}$ in the promoter region, the $P H B$ transcript was increased by $\mathrm{LiCl}$ in $\mathrm{HeLa}$ cells (Fig. 4A). These data indicated c-MYC-independent activation of $\mathrm{PHB}$ expression by Wnt signaling. To quantify the contributions of c-MYC and $\beta$-catenin on regulation of $P H B$ transcription, we analyzed transcript level of $P H B$ in Raji cells with treatment by 10058-F4, a c-myc inhibitor, that disrupts formation of the c-MYC/MAX heterodimer. Although $P H B$ expression was slightly repressed by $10058-\mathrm{F} 4$, Wnt signaling overcame this repression (Fig. 4B). These data indicate that Wnt signaling operates directly on the promoter of $P H B$.

\section{Discussion}

Although PHB has the potential to act as a tumor suppressor, antiproliferative protein and regulator of cell-cycle progression, recent studies have reported that overexpression of PHB was found in various tumor cells including various leukemic cells. Based on our proteomic analysis from AML patients, PHB level was greatly elevated (data not shown). Despite its role as a tumor suppressor, PHB level can be increased in phorbol ester-treated chronic leukemic B-lymphocytes (40). We suggested that development of AML or ALL can be associated with altered expression of specific mitochondrial proteins such as PHB, as shown in chronic leukemic B-lymphocytes. Many research projects reported the Wnt signal is required for the development of leukemic cells (41-43). The elevated PHB level in leukemic cells can be led by leukemic driven forces and we searched $\mathrm{PHB}$-inducing signals using promoter investigation (Fig. 3A).

We focused on the regulatory signals involved in $P H B$ expression that promote cell proliferation such as EGF and Wnt-mediated signals. In our data, $P H B$ could be expressed by Wnt signaling which is relevant in AML/ALL cells (32). AML translocation products, such a RUNX1-RUNX1T1, or epigenetic inactivation of pathway like Wnt inhibitory factor (WIF1) activate the Wnt pathway increasing TCF-4/ LEF-1-dependent transcription $(31,44)$. In the activated Wnt pathway of leukemic cells, PHB could be overexpressed via the TCF-4/LEF-1 binding motif in its promoter region (Fig. 3). Although it was reported that $P H B$ promoter had a c-MYC binding region and that the $\mathrm{PHB}$ co-localized with c-FOS and c-MYC $(25,45)$, our data showed that the role of c-MYC activity in expression of $P H B$ was less than $\beta$-catenin activity in leukemic cells (Fig. 4). Additionally although we assessed the role of a sequence similar to the E-box sequence located at -384 in the $P H B$ promoter, CACCTG, the response to $\mathrm{LiCl}$ 
of the mutant promoter in this region was similar to that of the region, -257 to -252 (data not shown). These data indicate that the -257 to -252 region is a major functional site of the c-MYC response to Wnt signaling.

The elevated level of PHB provides several arguments as to its role in tumorigenesis. Clearly, the expression was downregulated by growth signals (Fig. 1C) and this reinforces its role as a tumor suppressor. We analyzed the mRNA sequences in several leukemic cells. Although several mutations were found in some leukemic cells, most of their sequences were normal (data not shown). These data suggest that the increased transcription of $P H B$ in leukemic cells by Wnt signaling remains as yet unknown. PHB may be a common differentially expressed nuclear matrix protein in some tumor cells. PHB was distributed mainly in the regions of the nuclear membrane and cytoplasm in several cell types (45). Also PHB exists as a component of the nuclear matrix, and expression of $P H B$ was downregulated during differentiation $(27,46)$. In SK-N-SH cells, the expression of $P H B$ is relatively strong compared with retinoic acid-treated cells. By c-Jun-N-terminal kinase-1 (JNK-1) activity, phosphorylated PHB could suppress differentiation by inhibiting MEF2- and MRF-mediated gene transcription $(47,48)$.

In this study, we found that PHB has TCF-4 binding site in the promoter region and its expression is elevated by $\mathrm{LiCl}$. However, mutant 1 reporter without TCF-4 binding site was not affected by $\mathrm{LiCl}$ treatment and Fig. 3D shows that the accumulated $\beta$-catenin bound to $\mathrm{PHB}$ promoter region through the TCF-4/LEF-1 binding sequence (TCFbs1) in the treatment of $\mathrm{LiCl}$. Thus indicating that $P H B$ is one of the direct Wnt target genes. Although we found that $P H B$ is a direct target gene of Wnt signal pathway and it is overexpressed in acute leukemic cells, the effect of excess PHB remains unknown. Further studies are needed to determine the effects of PHB overexpression in leukemia.

\section{Acknowledgements}

This study was supported by the National Research Foundation of Korea (NRF) grant funded by the Korean Government (2013-R1A1A1007596 and 2015-M3A9C7030181). Also, this study was financially supported by Chonnam National University (grant no. 2012-0828).

\section{References}

1. McClung JK, Jupe ER, Liu XT and Dell'Orco RT: Prohibitin: Potential role in senescence, development, and tumor suppression. Exp Gerontol 30: 99-124, 1995.

2. Snedden WA and Fromm H: Characterization of the plant homologue of prohibitin, a gene associated with antiproliferative activity in mammalian cells. Plant Mol Biol 33: 753-756, 1997.

3. Loukas A and Maizels RM: Cloning and characterisation of a prohibitin gene from infective larvae of the parasitic nematode Toxocara canis. DNA Seq 9: 323-328, 1998.

4. Eveleth DD Jr and Marsh JL: Sequence and expression of the Cc gene, a member of the dopa decarboxylase gene cluster of Drosophila: Possible translational regulation. Nucleic Acids Res 14: 6169-6183, 1986.

5. Sato T, Saito H, Swensen J, Olifant A, Wood C, Danner D, Sakamoto T, Takita K, Kasumi F, Miki Y, et al: The human prohibitin gene located on chromosome $17 \mathrm{q} 21$ is mutated in sporadic breast cancer. Cancer Res 52: 1643-1646, 1992.
6. Back JW, Sanz MA, De Jong L, De Koning LJ, Nijtmans LG, De Koster CG, Grivell LA, Van Der Spek H and Muijsers AO: A structure for the yeast prohibitin complex: Structure prediction and evidence from chemical crosslinking and mass spectrometry. Protein Sci 11: 2471-2478, 2002.

7. Nijtmans LG, Artal SM, Grivell LA and Coates PJ: The mitochondrial PHB complex: Roles in mitochondrial respiratory complex assembly, ageing and degenerative disease. Cell Mol Life Sci 59: 143-155, 2002.

8. Sharma A and Qadri A: Vi polysaccharide of Salmonella typhi targets the prohibitin family of molecules in intestinal epithelial cells and suppresses early inflammatory responses. Proc Natl Acad Sci USA 101: 17492-17497, 2004.

9. Kolonin MG, Saha PK, Chan L, Pasqualini R and Arap W: Reversal of obesity by targeted ablation of adipose tissue. Nat Med 10: 625-632, 2004.

10. Rajalingam $\mathrm{K}$ and Rudel T: Ras-Raf signaling needs prohibitin. Cell Cycle 4: 1503-1505, 2005.

11. Mishra S, Murphy LC, Nyomba BL and Murphy LJ: Prohibitin: A potential target for new therapeutics. Trends Mol Med 11: 192-197, 2005.

12. Mishra S, Murphy LC and Murphy LJ: The prohibitins: Emerging roles in diverse functions. J Cell Mol Med 10: 353-363, 2006.

13. Wang S, Fusaro G, Padmanabhan J and Chellappan SP: Prohibitin co-localizes with $\mathrm{Rb}$ in the nucleus and recruits $\mathrm{N}-\mathrm{CoR}$ and HDAC1 for transcriptional repression. Oncogene 21: 8388-8396, 2002.

14. Fusaro G, Dasgupta P, Rastogi S, Joshi B and Chellappan S: Prohibitin induces the transcriptional activity of p53 and is exported from the nucleus upon apoptotic signaling. J Biol Chem 278: 47853-47861, 2003.

15. Wang S, Nath N, Adlam M and Chellappan S: Prohibitin, a potential tumor suppressor, interacts with RB and regulates E2F function. Oncogene 18: 3501-3510, 1999.

16. Joshi B, Ko D, Ordonez-Ercan D and Chellappan SP: A putative coiled-coil domain of prohibitin is sufficient to repress E2F1mediated transcription and induce apoptosis. Biochem Biophys Res Commun 312: 459-466, 2003.

17. Wang S, Zhang B and Faller DV: BRG1/BRM and prohibitin are required for growth suppression by estrogen antagonists. EMBO J 23: 2293-2303, 2004.

18. DiRenzo J, Shang Y, Phelan M, Sif S, Myers M, Kingston R and Brown M: BRG-1 is recruited to estrogen-responsive promoters and cooperates with factors involved in histone acetylation. Mol Cell Biol 20: 7541-7549, 2000.

19. He B, Feng Q, Mukherjee A, Lonard DM, DeMayo FJ, Katzenellenbogen BS, Lydon JP and O'Malley BW: A repressive role for prohibitin in estrogen signaling. Mol Endocrinol 22: 344-360, 2008

20. Artal-Sanz M, Tsang WY, Willems EM, Grivell LA, Lemire BD, van der Spek $\mathrm{H}$ and Nijtmans LG: The mitochondrial prohibitin complex is essential for embryonic viability and germline function in Caenorhabditis elegans. J Biol Chem 278: 32091-32099, 2003.

21. Arraztoa JA, Zhou J, Marcu D, Cheng C, Bonner R, Chen M, Xiang C, Brownstein M, Maisey K, Imarai M, et al: Identification of genes expressed in primate primordial oocytes. Hum Reprod 20: 476-483, 2005.

22. Coates PJ, Nenutil R, McGregor A, Picksley SM, Crouch DH, Hall PA and Wright EG: Mammalian prohibitin proteins respond to mitochondrial stress and decrease during cellular senescence. Exp Cell Res 265: 262-273, 2001.

23. Ryu JW, Kim HJ, Lee YS, Myong NH, Hwang CH, Lee GS and Yom HC: The proteomics approach to find biomarkers in gastric cancer. J Korean Med Sci 18: 505-509, 2003.

24. Xu Z, Wu J and Zha X: Up-regulation of prohibitin 1 is involved in the proliferation and migration of liver cancer cells. Sci China Life Sci 54: 121-127, 2011.

25. Menssen A and Hermeking H: Characterization of the c-MYCregulated transcriptome by SAGE: Identification and analysis of c-MYC target genes. Proc Natl Acad Sci USA 99: 6274-6279, 2002.

26. Fusaro G, Wang S and Chellappan S: Differential regulation of $\mathrm{Rb}$ family proteins and prohibitin during camptothecin-induced apoptosis. Oncogene 21: 4539-4548, 2002.

27. Li QF, Liang Y, Shi SL, Liu QR, Xu DH, Jing GJ, Wang SY and Kong HY: Localization of prohibitin in the nuclear matrix and alteration of its expression during differentiation of human neuroblastoma SK-N-SH cells induced by retinoic acid. Cell Mol Neurobiol 31: 203-211, 2011. 
28. Gilliland DG, Jordan CT and Felix CA: The molecular basis of leukemia, Hematology Am Soc Hematol Educ Progam 2004: 80-97, 2004.

29. Hu Y, Chen Y, Douglas L and Li S: Beta-catenin is essential for survival of leukemic stem cells insensitive to kinase inhibition in mice with BCR-ABL-induced chronic myeloid leukemia. Leukemia 23: 109-116, 2009.

30. Zhao C, Blum J, Chen A, Kwon HY, Jung SH, Cook JM, Lagoo A and Reya T: Loss of beta-catenin impairs the renewal of normal and CML stem cells in vivo. Cancer Cell 12: 528-541, 2007.

31. Müller-Tidow C, Steffen B, Cauvet T, Tickenbrock L, Ji P, Diederichs S, Sargin B, Köhler G, Stelljes M, Puccetti E, et al: Translocation products in acute myeloid leukemia activate the Wnt signaling pathway in hematopoietic cells. Mol Cell Biol 24: 2890-2904, 2004.

32. Siapati EK, Papadaki M, Kozaou Z, Rouka E, Michali E, Savvidou I, Gogos D, Kyriakou D, Anagnostopoulos NI and Vassilopoulos G: Proliferation and bone marrow engraftment of AML blasts is dependent on $\beta$-catenin signalling. $\mathrm{Br} \mathrm{J}$ Haematol 152: 164-174, 2011.

33. Gururajan M, Chui R, Karuppannan AK, Ke J, Jennings CD and Bondada S: c-Jun N-terminal kinase (JNK) is required for survival and proliferation of B-lymphoma cells. Blood 106: 1382-1391, 2005

34. Artal-Sanz M and Tavernarakis N: Opposing function of mitochondrial prohibitin in aging. Aging (Albany, NY) 2: 1004-1011, 2010.

35. Yu K, Ravera CP, Chen YN and McMahon G: Regulation of Myc-dependent apoptosis by p53, c-Jun N-terminal kinases/ stress-activated protein kinases, and $\mathrm{Mdm}-2$. Cell Growth Differ 8: 731-742, 1997.

36. Noguchi K, Yamana H, Kitanaka C, Mochizuki T, Kokubu A and Kuchino Y: Differential role of the JNK and p38 MAPK pathway in c-Myc- and s-Myc-mediated apoptosis. Biochem Biophys Res Commun 267: 221-227, 2000

37. Jauregui MP, Sanchez SR, Ewton AA, Rice L, Perkins SL, Dunphy $\mathrm{CH}$ and Chang CC: The role of beta-catenin in chronic myeloproliferative disorders. Hum Pathol 39: 1454-1458, 2008.
38. Jost E, Gezer D, Wilop S, Suzuki H, Herman JG, Osieka R and Galm O: Epigenetic dysregulation of secreted Frizzled-related proteins in multiple myeloma. Cancer Lett 281: 24-31, 2009.

39. Stambolic V, Ruel L and Woodgett JR: Lithium inhibits glycogen synthase kinase- 3 activity and mimics wingless signalling in intact cells. Curr Biol 6: 1664-1668, 1996.

40. Woodlock TJ, Bethlendy G and Segel GB: Prohibitin expression is increased in phorbol ester-treated chronic leukemic B-lymphocytes. Blood Cells Mol Dis 27: 27-34, 2001.

41. Wang Y, Krivtsov AV, Sinha AU, North TE, Goessling W, Feng Z, Zon LI and Armstrong SA: The Wnt/beta-catenin pathway is required for the development of leukemia stem cells in AML. Science 327: 1650-1653, 2010.

42. Kim Y, Thanendrarajan S and Schmidt-Wolf IGH: Wnt/ $\beta$-catenin: A new therapeutic approach to acute myeloid leukemia, Leuk Res Treatment 2011: 428960, 2011

43. Ashihara E, Takada T and Maekawa T: Targeting the canonical Wnt/ $\beta$-catenin pathway in hematological malignancies. Cancer Sci 106: 665-671, 2015.

44. Chim CS, Chan WW, Pang A and Kwong YL: Preferential methylation of Wnt inhibitory factor-1 in acute promyelocytic leukemia: An independent poor prognostic factor. Leukemia 20: 907-909, 2006

45. Xu DH, Tang J, Li QF, Shi SL, Chen XF and Liang Y: Positional and expressive alteration of prohibitin during the induced differentiation of human hepatocarcinoma SMMC-7721 cells. World J Gastroenterol 14: 5008-5014, 2008.

46. Zhao $\mathrm{CH}$ and Li QF: Altered profiles of nuclear matrix proteins during the differentiation of human gastric mucous adenocarcinoma MGc80-3 cells. World J Gastroenterol 11: 4628-4633, 2005.

47. Feng W, Webb P, Nguyen P, Liu X, Li J, Karin M and Kushner PJ: Potentiation of estrogen receptor activation function 1 (AF-1) by Src/JNK through a serine 118-independent pathway. Mol Endocrinol 15: 32-45, 2001.

48. Sun L, Liu L, Yang XJ and Wu Z: Akt binds prohibitin 2 and relieves its repression of MyoD and muscle differentiation. J Cell Sci 117: 3021-3029, 2004 